\title{
Phase retrieval and compression of low-power white-light pulses
}

\author{
D. Wegkamp ${ }^{1}$, D. Brida 2 , S. Bonora ${ }^{3}$, G. Cerullo ${ }^{2}$, J. Stähler ${ }^{1}$, M. Wolf1 and S. Wall1,* \\ ${ }^{1}$ Fritz-Haber-Institut der Max-Planck-Gesellschaft, Department of Physical Chemistry, Berlin, Germany \\ ${ }^{2}$ IFN-CNR, Dipartimento di Fisica, Politecnico di Milano, Milano, Italy \\ IIFN-CNR, D.E.I., Università di Padova, Padova, Italy
}

\begin{abstract}
We characterize and compress sub-nJ visible white-light continuum (WLC) pulses generated by self-phase modulation in yttrium aluminium garnet. The spectral phase is retrieved by spectrally resolving the transient reflectivity from an optically-excited transition metal oxide. This measured phase is compensated by applying the appropriate distortion to a deformable mirror. By comparing the response of two different materials we show that the white-light pulses can be compressed to approximately $10 \mathrm{fs}$ duration.
\end{abstract}

The use of ultra-broadband pulses generated via self-phase modulation has become an important tool in physics and chemistry ${ }^{1,2}$. The large frequency content of these pulses can be exploited to measure temporal dynamics over a broad frequency range simultaneously, drastically reducing acquisition times and increasing experimental information. Additionally, the increased bandwidth supports pulse durations significantly shorter than the generating pulse, improving the temporal resolution. However, a key pre-requisite for the application of these pulses is an accurate measurement and control of their spectral phase so that, through suitable dispersive delay lines (pulse compressors) that adjust the relative group delay (GD) of the different frequency components of the pulse, a near transform-limited (TL) pulse can be achieved.

One of the simplest and most powerful methods of generating broadband WLC pulses is by focusing a $\mu \mathrm{J}$-level energy pulse in a bulk transparent material ${ }^{3,4}$. A complex interplay between numerous nonlinear effects, including temporal self-phase modulation, spatial self-focusing, filamentation and plasma formation ${ }^{5}$ results in a broad, smooth and stable spectrum, making it an ideal source for broadband spectroscopy. However, the nature of the WLC generation mechanism results in long pulses with significant temporal chirp, which is challenging to correct over a broad spectral range. In addition, pulse energies are low, with typical spectral energy densities of $10 \mathrm{pJ} / \mathrm{nm}^{6}$, making their temporal characterization difficult. Many nonlinear optical techniques, such as frequency-resolved optical gating ${ }^{7}$, nonlinear spectral interferometry ${ }^{8}$ and their variants ${ }^{9}$, can be used for full characterization in amplitude and phase of ultrashort light pulses. However the application of these methods to WLC pulses is difficult, due to their ultrabroad bandwidth, which requires

*Email: wall@fhi-berlin.mpg.de 
characterization schemes that make use of very thin nonlinear crystals, and their low peak power, which results in weak nonlinear signals.

In this work we present a fast and simple technique for measuring and compensating for the frequency chirp of low-power, visible WLC pulses. We characterize the WLC by measuring the spectrally-resolved transient reflectivity ${ }^{10}$ of $\mathrm{La}_{0.5} \mathrm{Sr}_{1.5} \mathrm{MnO}_{4}$ (LSMO) after excitation with $800 \mathrm{~nm}$ ultrashort pulses. From the frequency resolved transients, we retrieve the GD and thus the spectral phase, which is compensated by applying an appropriate deformation to a calibrated deformable mirror, compressing the pulse close to the TL. Due to the large photo-response of LSMO over a broad frequency range in the visible spectral region, pulses can be characterized and compressed, in situ, within a few minutes. Importantly, we show that the phase measured using LSMO is accurate by comparing the response to that obtained with a different material.

Figure 1(a) shows the experimental setup used to generate, characterize and compress the WLC pulses. The $700 \mathrm{~mW}$ output of a $40 \mathrm{fs}, 800 \mathrm{~nm}$ Ti:sapphire laser, operating at $297 \mathrm{kHz}$, was split into a high energy pump beam and a WLC probe. Stable and broadband WLC was generated by focussing $800 \mathrm{~nm}$ pulses into a $2 \mathrm{~mm}$ yttrium aluminium garnet crystal and optimized using a neutral density filter and iris (Fig. 1(b)). The WLC was collimated by a $50 \mathrm{~mm}$ off-axis parabola and apertured to approximately $1 \mathrm{~mm}$ in diameter. The WLC was compressed in a zero-dispersion pulse shaper with the deformable mirror in the Fourier plane. The pulse shaper was in a $4 f$ configuration consisting of a grating $\left(600 \mathrm{~g} / \mathrm{mm}\right.$ plane ruled grating with $8.6^{\circ}$ blaze) and spherical mirror $(f=250 \mathrm{~mm})$. After compression, the WLC was focused onto the sample and overlapped with the $800 \mathrm{~nm}$ pump pulse.

Recently, the phase of broadband pulses generated in an optical fiber has been retrieved by spectrally resolving the photoinduced change in reflectivity of $\mathrm{VO}_{2}$ at $\mathrm{IR}$ wavelengths ${ }^{10}$. In these measurements the pump pulse modulates the reflectivity of the material, which affects different wavelengths at different times depending on the GD of the probe pulse, and the retrieved phase was found to be in good agreement with that obtained by standard pulse characterization techniques. Transient reflectivity measurements have several advantages over conventional pulse characterization techniques. Principally, the alignment is straightforward with no phase matching issues, and the measurements are performed in reflection, allowing the same apparatus to be used for both characterization and pump-probe measurements. However, the material must satisfy two conditions: the magnitude of the pump-induced reflectivity change must be non-vanishing over the spectral content of the probe pulse and the temporal response should be wavelength independent, 
rising with the integral of the pump pulse. This is a particularly challenging requirement for probe energies higher than the pump pulse. In semiconductors, the pump changes the electronic population within a static band structure, which only has a small effect on transitions at the higher energies. However, for strongly correlated electron materials (SCEMs), large changes in the reflectivity occur over a broad bandwidth due to the strong (eV-scale) interactions between the electrons. In these materials, photoexcitation changes the band structure ${ }^{11}$, and thus the density of states which is probed through the reflectivity of the WLC. These processes are illustrated in Fig. $1(\mathrm{c})$.

Amongst SCEMS, manganites represent an ideal choice for characterizing visible pulses as they contain broad resonances at visible wavelengths that are strongly modified by photoexcitation. We chose LSMO as it exhibits large photo-induced reflectivity changes in the visible region ${ }^{12}$, even when pumped with substantially lower energy photons ${ }^{13}$, due to a broad charge transfer resonance that is particularly sensitive to the $\mathrm{Mn}$ charge distribution. In addition, at room temperature no photoinduced phase transitions, which may complicate the optical response ${ }^{14}$, occur.

Figure 2(a) shows the time and frequency resolved transient reflectivity of LSMO probed by WLC, with no distortion applied to the deformable mirror. Spectra were recorded with 100 ms integration time and 5 fs time-steps using an optical multichannel analyser (OMA). A large-amplitude, low-noise response was observed across the entire WLC spectrum, ranging from $-10 \%$ to $+3 \%$ for a modest pump fluence of $1.8 \mathrm{~mJ} \mathrm{~cm}^{-2}$. The change of sign in the transient reflectivity is due to the shift and broadening of the charge transfer resonance induced by the pump pulse.

The time-zero response shows a parabolic-like frequency dependence, demonstrating that the spectral phase of the WLC after the pulse shaper is dominated by $3^{\text {rd }}$ order, and higher, terms. We extract the frequency dependent GD, $\tau_{G}(v)$, by fitting an error function, with a rise time set by the integral of our pulse duration at each frequency, $v$, from which we extract the half-rise time of the transient response (see Fig. 2d). The dashed line in Fig. 2(a) shows the GD from which, the spectral phase is obtained from $\phi(v)=\int \tau_{G}(v) d v$. Using a calibrated deformable mirror ${ }^{15}$, we apply the appropriate distortion to compensate for this phase and achieve a compressed pulse. Figure 2(b) shows the transient reflectivity for a compressed WLC pulse after only two iterations. With the final deformation, a flat GD is obtained indicating that the pulse is close to the TL. 
We retrieve the temporal pulse envelope by the inverse Fourier transform $I(t) \propto\left|\int_{0}^{\infty} \sqrt{I(v)} e^{i \phi(v)} e^{-2 \pi i v t} d v\right|^{2}$, where $v$ is the frequency and $I(v)$ is the WLC spectral intensity. Figure 2(c) shows $I(t)$ for the phase extracted from the uncompressed and compressed pulses, together with the TL $(\phi(v)=$ const.). The TL and compressed pulses have full-width halfmaxima of $10.5 \mathrm{fs}$ and $11.7 \mathrm{fs}$ respectively, demonstrating that the pulse is well compressed. The uncompressed pulse is highly asymmetric and is significantly longer. An $11.7 \mathrm{fs}$ pulse can be easily measured, even though the gating pulse which excites the system is much longer ( $40 \mathrm{fs}$ ) because the accuracy of the GD is only limited by the determination of the half rise point. As the transient signal of LSMO is large compared to the noise, this can be determined with a precision of better than $\sigma= \pm 5$ fs at all wavelengths, as shown in Fig $2 \mathrm{~d}$. As the spectral phase is obtained by integrating the GD over frequency, it is robust to random point-to-point fluctuations in the measured GD. By applying Gaussian noise with a $5 \mathrm{fs}$ variance to our extracted GD values, we find that the retrieved pulse duration is practically unaffected.

The above analysis relies on the assumption that the onset of the reflectivity transient is independent of wavelength, i.e. $R\left(v, t_{p}\right)=R(v)+\Delta R(v) \Theta\left(t_{p}\right)$, where $R\left(v, t_{p}\right)$ is the transient reflectivity measured by the probe pulse and at a time $t_{p}$ after the pump pulse. $\Delta R(v)$ is the pump induced change which varies with probe wavelength. However, $\Theta\left(t_{p}\right)$ is a step-like profile of the initial transient, which should be independent of the wavelength. If the response at certain wavelengths is delayed, i.e. $\Theta$ depends on wavelength due to some bottleneck processes, then the extracted phase will incorporate the material response. Therefore, in order to check the validity of the compression using LSMO, we compare it to the response obtained from another SCEM, $\mathrm{VO}_{2}$.

At high excitation fluences $\mathrm{VO}_{2}$ exhibits a photoinduced phase transition, which may influence the extracted $G D^{16}$. Therefore, here we focus on the low-fluence regime. Figure 3(a) shows the spectrally resolved transient reflectivity of $\mathrm{VO}_{2}$ under the same excitation conditions as those used for LSMO. The reflectivity change is lower, $<1 \%$, requiring longer acquisition times, but coherent optical phonons are observed, as indicated by the line out in Fig $3(\mathrm{~b})$. The compressed WLC probe is the same as that used for LSMO in Fig 2(b). As the phase of optical phonons is locked by the pump pulse and is thus independent of the probe wavelength ${ }^{17}$, we use the phonon phase to extract the GD of the WLC pulse. This is shown as the dashed line in Fig. 3(a), and should be compared with the phase extracted from LSMO, reproduced in Fig. 3(a) as the solid line but shifted by one phonon period. 
These measurements are in excellent agreement and validate the use of LSMO for phasecharacterisation purposes.

To summarize, we have presented a simple method to measure and compress broadband lowenergy WLC pulses to approximately $10 \mathrm{fs}$ duration and demonstrated that the transient reflectivity of LSMO provides an accurate measurement of the WLC spectral phase. The experimental geometry used is amenable to pump-probe experiments as it can be performed in situ and can easily be implemented inside a cryostat or ultra high vacuum environments, where the deformable mirror compressor can easily compensate for additional chirp arising from entrance windows. The ease of these measurements means that pulse characterization can be conveniently performed before carrying out the experiment. By turning the grating, different wavelength ranges can be selected, extending the probe spectral range into the UV or IR. Once compressed, spectroscopic information can be sacrificed for improved signal-to-noise with lock-in detection, without compromising temporal resolution. We anticipate the use of these pulses in time- and energy-resolved electronic sum-frequency generation at interfaces, surfaces and magnetic materials ${ }^{18}$.

We gratefully thank John C. Mitchell and Richard Haglund for providing samples. S.W. acknowledges support from the Alexander von Humboldt Foundation. 


\section{Figures}

(a)

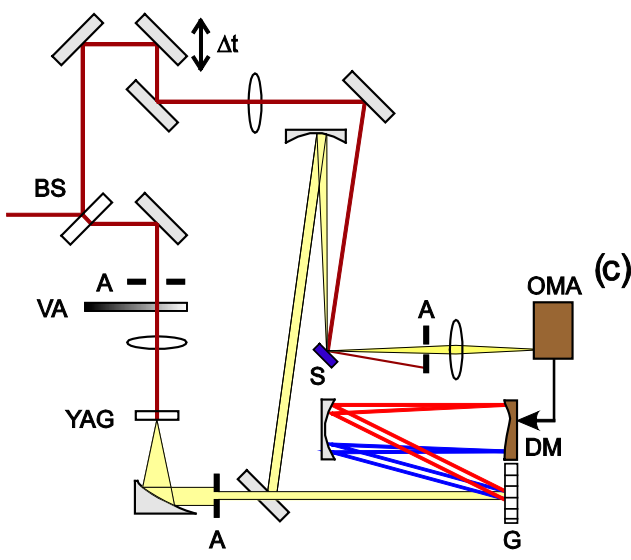

(b)

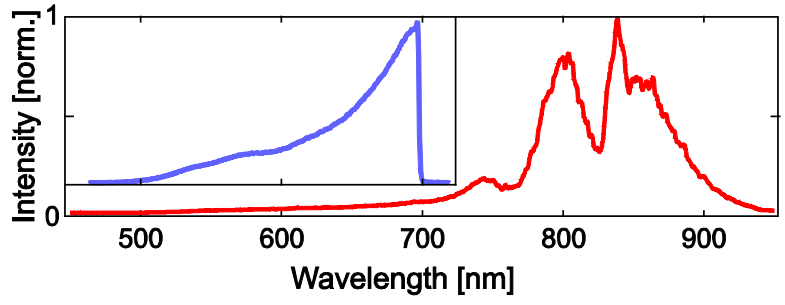

(c)

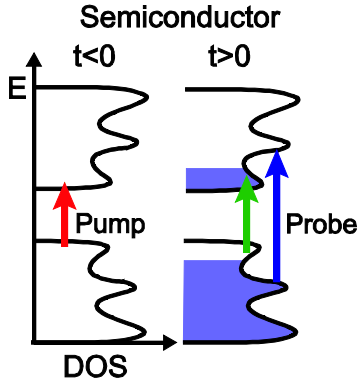

SCEM

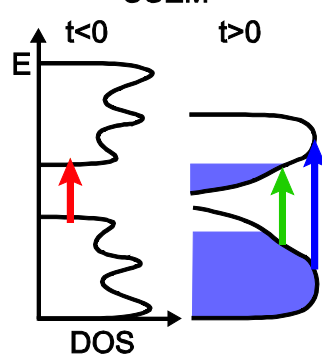

Figure 1: a. Schematic of WLC generation and characterization. BS - Beam Splitter, A Aperture, VA - Variable Attenuator, G - Grating, DM - Deformable Mirror, $\Delta \mathrm{t}$ - variable delay stage, OMA - Optical multichannel analyser. b. WLC spectrum measured before the grating. The inset shows the spectrum selected by the deformable mirror, at the sample surface. c. Schematic representation of the pump-induced effects on the reflectivity resulting from changes in the density of states (DOS) for a semiconductor and correlated material.
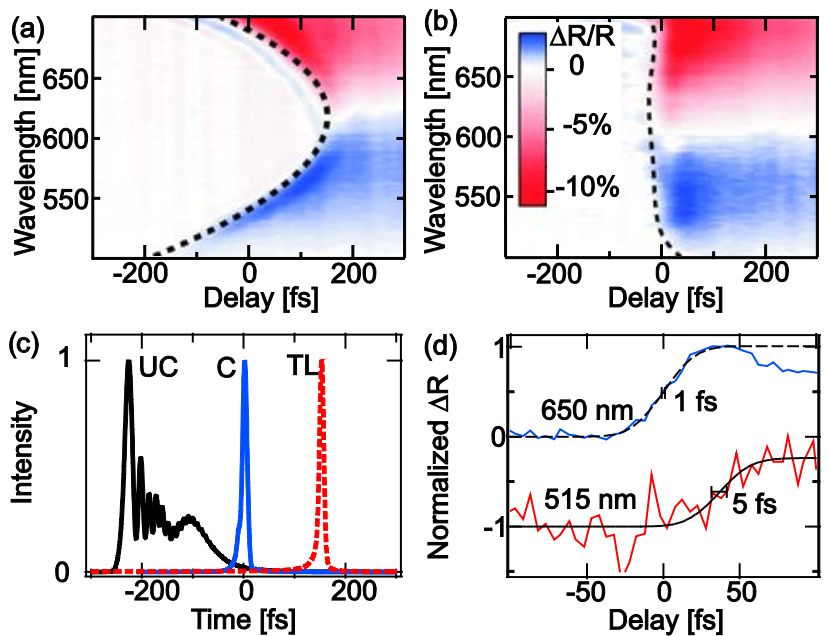

Figure 2: Time and frequency resolved transient reflectivity of LSMO measured with (a) the uncompressed WLC pulse and (b) the compressed pulse. Black dashed lines indicate extracted group delay. (c) Retrieved WLC intensity envelopes for uncompressed (UC), compressed (C), and transformlimited (TL) pulses. (d) Line-outs of the normalized transient reflectivity signal at $650 \mathrm{~nm}$ and $515 \mathrm{~nm}$ together with error function fits (dashed lines). The horizontal bars, of width $2 \sigma$, indicate the error in determining the half-rise point at each wavelength. 


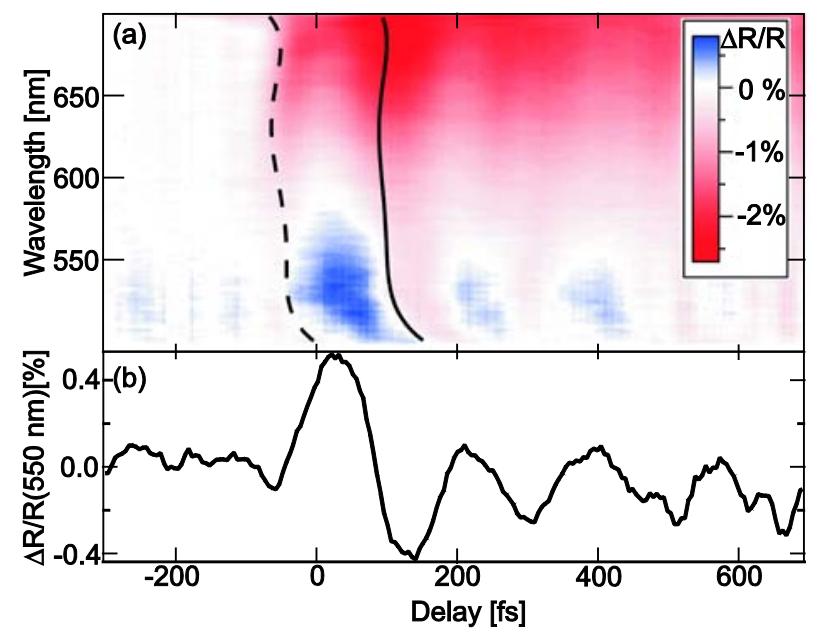

Figure 3: (a) Time and frequency resolved transient reflectivity of $\mathrm{VO}_{2}$. (b) Lineout of the reflectivity at $550 \mathrm{~nm}$ (with $20 \mathrm{~nm}$ bandwidth). The dashed line is the group delay extracted by fitting the phase offset of the phonon for each wavelength. The solid line reproduces the phase obtained from LSMO (shifted by one phonon period). 


\section{References}

${ }^{1}$ U. Megerle, I. Pugliesi, C. Schriever, C.F. Sailer, E. Riedle, Appl Phys B 96, 215 (2009)

2 D. Polli, P. Altoè, O. Weingart, K. M. Spillane, C. Manzoni, D. Brida, G. Tomasello, G. Orlandi, P. Kukura, R. A. Mathies, M. Garavelli, G. Cerullo, Nature 467, 440 (2010)

${ }^{3}$ R. R. Alfano, and S. L. Shapiro, Phys. Rev. Lett. 24, 584 (1970)

${ }^{4}$ R.R. Alfano, The Supercontinuum Laser Source (Springer, Berlin, 2006)

${ }^{5}$ A.L. Gaeta, Phys. Rev. Lett. 84, 3582 (2000)

${ }^{6}$ M. Bradler, P. Baum, and E. Riedle, Appl. Phys. B 97, 561 (2009)

${ }^{7}$ D.J. Kane and R. Trebino, IEEE J. Quantum Electron. 29, 571, 1993

${ }^{8}$ C. Iaconis and I. A. Walmsley, Opt. Lett. 23, 792 (1998)

${ }^{9}$ I.A. Walmsley and C. Dorrer, Adv. Opt. Photon. 1, 308 (2009)

${ }^{10}$ F. Cilento, C. Giannetti, G. Ferrini, S. Dal Conte, T. Sala, G. Coslovich, M. Rini, A. Cavalleri, F. Parmigiani, Appl. Phys. Lett. 96, 021102 (2010)

${ }^{11}$ F. Schmitt, P. S. Kirchmann, U. Bovensiepen, R. G. Moore, L. Rettig, M. Krenz, J.-H. Chu, N. Ru, L. Perfetti, D. H. Lu, M. Wolf, I. R. Fisher, Z.-X. Shen, Science 321, 1649 (2008)

${ }^{12}$ T. Ogasawara, T. Kimura, T. Ishikawa, M. Kuwata-Gonokami, Y. Tokura, Phys. Rev. B 63, 113105 (2001)

${ }^{13}$ R. I. Tobey, R. Prabakharan, A. T. J. Boothroyd, A. Cavalleri, Phys. Rev. Lett. 101, 197404 (2008)

${ }^{14}$ D. Polli, M. Rini, S. Wall, R.W Schoenlein, Y. Tomioka, Y. Tokura, G. Cerullo, A. Cavalleri Nature Materials 6, 643 (2007)

${ }^{15}$ S. Bonora, I. Capraro, L. Poletto, M. Romanin, C. Trestino, and P. Villoresi, Rev. Sci. Instrum. 77, 093102 (2006)

${ }^{16}$ A. Cavalleri, Th. Dekorsy, H. Chong, J.C. Kieffer, R.W. Schoenlein Phys. Rev. B 70, 161102(R) (2004)

${ }^{17}$ H. J. Zeiger, J. Vidal, T. K. Cheng, E. P. Ippen, G. Dresselhaus, M. S. Dresselhaus, Phys. Rev. B 45, 768 (1992)

${ }^{18}$ K. Sekiguchi, S. Yamaguchi, T. Tahara, J. Chem. Phys 128, 114715 (2008) 